\title{
INFLUENCIA DE LA ESPIRITUALIDAD EN LAS DIMENSIONES DEL CUIDADO EN MUJERES ADULTAS MAYORES ${ }^{1}$
}

\author{
INFLUENCE OF SPIRITUALITY ON THE DIMENSIONS OF \\ CAREGIVING AMONG ELDERLY WOMEN
}

\section{INFLUENCIA DA ESPIRITUALIDADE NAS DIMENSÓES DO CUIDADO EM MULHERES IDOSAS}

\author{
Fabiola Castellanos Soriano* \\ MaUricio Rincón Andrade**
}

\section{RESUMEN}

Objetivo: Describir la influencia de la espiritualidad en las dimensiones del cuidado en mujeres adultas mayores de un centro día de la ciudad de Bogotá. Material y Método: Estudio cualitativo etnográfico con entrevistas a profundidad, diarios de campo y observación participante durante actividades de arteterapia y cartas al cuerpo. Participaron 12 mujeres mayores de 60 años que asistían a un programa de atención en modalidad día en la ciudad de Bogotá (Colombia). Los datos fueron analizados siguiendo las cuatro fases propuestas por Leininger. Se tuvieron en cuenta los criterios éticos de la Resolución 8430 del Ministerio de Salud y la Declaración de Helsinki. Resultados: En la dimensión física se evidenciaron prácticas que integran capacitación constante, alimentación y ejercicio. En la dimensión psicológica se reflejaron necesidad de contar con relaciones sociales, contacto con la música y la naturaleza, así como emociones positivas. En la dimensión espiritual se encontró que está influenciada por la creencia de un ser superior, ayudar a otros y el constante agradecimiento. Conclusiones: La espiritualidad, en las mujeres participantes de la investigación, impregna la dimensión física como la psicológica, por ello se requiere implementar procesos de atención en salud integrales que reivindiquen estas prácticas para fortalecer el cuidado y el envejecimiento saludables.

Palabras clave: Cuidado; Espiritualidad; Envejecimiento; Adulto mayor.

\begin{abstract}
Objective: To describe the influence of spirituality on the dimensions of caregiving among elderly women in a day care center in the city of Bogotá (Colombia). Material and Method: Qualitative ethnographic study carried out using in-depth interviews, field diaries and participant observation during art therapy activities and letters

\footnotetext{
${ }^{1}$ Financiamiento: Convocatoria Interna 00006898 Vicerrectoría de Investigación, Pontificia Universidad Javeriana, Bogotá, Colombia.

*Doctora en Enfermería. Facultad de Enfermería, Pontificia Universidad Javeriana, Bogotá, Colombia. Líder del Grupo conceptualización y práctica de Enfermería. ORCID: https://orcid.org/0000-0002-0509-3096 Email: fabiola.castellano@ javeriana.edu.co Autora de correspondencia.

** Doctor en Teología. Facultad de Teología de la Pontificia Universidad Javeriana, Bogotá, Colombia. ORCID: https://orcid. org/0000-0002-9776-3519 Email: mauricio-rincon@javeriana.edu.co
} 
to the body. Twelve women over 60 years who attended a day care program in the city of Bogotá participated. The data were analyzed following the four phases proposed by Leininger. The ethical criteria of Resolution 8430 of the Ministry of Health and the Helsinki Declaration were also considered. Results: The study showed practices, in the physical dimension, that integrate constant training, food and exercise. In the psychological dimension, there was evidence for the need for social relationships, contact with music and nature, as well as positive emotions. The spiritual dimension was found to be influenced by the belief in a higher being, helping others, and constant gratitude. Conclusions: Spirituality, in the women participating in the research, influences the physical as well as the psychological dimension. It is, therefore, necessary to implement comprehensive health care processes that promote these practices to strengthen care and healthy aging.

Key words: Care; Spirituality; Aging; Elderly.

\section{RESUMO}

Objetivo: Descrever a influência da espiritualidade nas dimensóes do cuidado em mulheres idosas em uma creche na cidade de Bogotá (Colômbia). Material e Método: Estudo etnográfico qualitativo com entrevistas em profundidade, diários de campo e observaçáo dos participantes durante atividades de arte-terapia e cartas ao corpo. Participaram doze mulheres com mais de 60 anos de idade que frequentavam um programa de creche na cidade de Bogotá. Os dados foram analisados seguindo as quatro fases propostas por Leininger. Foram considerados os critérios éticos da Resolução 8430 do Ministério da Saúde e da Declaraçáo de Helsinque. Resultados: Na dimensão física, evidenciaram-se práticas que integram treinamento constante, alimentaçáo e exercício físico. $\mathrm{Na}$ dimensão psicológica, houve evidência da necessidade de relacionamento social, contato com a música e a natureza, assim como as emoções positivas. $\mathrm{Na}$ dimensão espiritual, descobriu-se que é influenciada pela crença em um ser superior, a ajuda aos outros e gratidáo constante. Conclusóes: A espiritualidade, nas mulheres participantes da pesquisa, influencia tanto a dimensão física quanto a psicológica. Portanto, é necessária a implementação de processos de atenção integral à saúde que justifiquem essas práticas para fortalecer o cuidado e o envelhecimento saudáveis.

Palavras-chave: Cuidado; Espiritualidade; Envelhecimento; Idosos.

Fecha de recepción: 21/08/20

\section{INTRODUCCIÓN}

Los indicadores de transición demográfica mundial reflejan un generalizado envejecimiento de la población ${ }^{(1)}$. En Colombia se viene detectando un crecimiento superior en los adultos mayores comparado con la población en general. Las estimaciones para el 2020 reportaron un incremento del 3,76\%. Este crecimiento acelerado en la población mayor de 60 años se explica, entre otras razones, por la disminución de la fecundidad y la reducción en la mortalidad ${ }^{(2)}$.

Frente a este panorama se conectan otras condiciones que presentan los adultos mayores como: la discapacidad, aparición de enfermedades crónicas, deterioro en la salud mental, abandono y condiciones económicas precarias ${ }^{(3,4)}$. Esta con-
Fecha de aceptación: 20/04/2021

dición demográfica y sus implicaciones sugieren ofrecer cuidados culturalmente competentes, que reconozcan aquellos procesos socioculturales inmersos en el cuidado de la salud de los adultos mayores, sus cuidadores y sus familias. Además, identificar las áreas que fortalecen el cuidado que permiten alcanzar bienestar, confianza, autonomía y calidad de vida ${ }^{(5-7)}$.

El cuidado de los adultos mayores es permeado por creencias, valores, estereotipos, estructuras sociales y prácticas populares, que determinan las diversas maneras en que se activan y se movilizan diferentes grupos poblacionales para afrontar las exigencias de cuidado ${ }^{(8,9)}$.

En cuanto al cuidado de las mujeres adultas mayores, la literatura ha visibilizado en algunos estudios $^{(10,11)}$ una mayor participación de estas en 
procesos formativos de autocuidado y promoción de la salud, comparado con los hombres, argumentándose que las mujeres adultas mayores poseen mayor disposición para participar de estos procesos, así como una mayor apropiación de hábitos saludables.

Por su parte, la espiritualidad, como parte del cuidado es entendida como una dimensión fundamental en la realización humana, tácita, enmarcada en la integralidad de las personas, que se configura en un espacio cotidiano de la vida ${ }^{(12)}$, que provee bienestar y percepción de mejoramiento ante situaciones adversas ${ }^{(13,14)}$ y como una experiencia de lo divino ${ }^{(13)}$, que adquiere relevancia.

Estudiar la espiritualidad en el contexto de la salud y el cuidado implica descender al descubrimiento de espacios esenciales acerca de lo que se entiende por la vida, las metas de existir, las comprensiones acerca de la fe, la esperanza, el amor y la muerte, desde las diferentes dimensiones que esta puede llegar a tener en las personas ${ }^{(15-18)}$.

Considerando la necesidad de un cuidado culturalmente competente, que dialogue con la diversidad social actual y sus necesidades, este estudio visibiliza en un grupo de mujeres adultas mayores, los vínculos de la espiritualidad con las dimensiones del cuidado, con el fin de aportar a los procesos de consolidación de políticas públicas, atención interdisciplinaria en salud y reivindicación de las prácticas populares del cuidado en una sociedad que envejece.

\section{MATERIAL Y MÉTODO}

Se trata de un estudio cualitativo direccionado a la obtención de detalles complejos y exhaustivos de algunos fenómenos ${ }^{(19)}$, de tipo etnográfico, dado que permite adentrarse al fenómeno estudiado de manera holística, explorando la subjetividad y el contexto $^{(20)}$. La muestra de tipo intencional estuvo conformada por 12 mujeres, participantes de un programa de atención en modalidad centro día, de una caja de compensación en la ciudad de Bogotá. A este programa para el adulto mayor asisten alrededor de 80 personas, en su mayoría mujeres.

Se tuvo en cuenta como criterios de inclusión: personas mayores de 60 años, con funciones mentales preservadas, como atención, memoria, pensamiento, comprensión y fluidez verbal; no se consideró para participar en el estudio la funcionalidad física o grado de dependencia. Estas funciones mentales las corrobora el centro día en el ingreso, dado que es un programa para adultos mayores funcionales $\mathrm{y}$ con funciones mentales preservadas.

Los métodos para la obtención de la información fueron: observación participante durante actividades que se implementaron, como el taller de arteterapia, forma de terapia expresiva que usa el proceso creativo del arte para ayudar a las personas a comunicarse mejor, reducir el estrés y superar problemas emocionales. En esta investigación se utilizó el taller de siluetas y los mandalas. Además, se les entregó un formato llamado "Carta al cuerpo", en donde cada participante le escribió una carta a su propio cuerpo. Se trabajaron entrevistas en profundidad y diarios de campo.

Las entrevistas tuvieron una duración aproximada de 40 minutos en promedio; fueron grabadas, teniendo en cuenta que los participantes firmaron consentimiento informado, respetándose su privacidad y anonimato. Las preguntas eje de indagación en las entrevistas se enfocaron en la narración de experiencias acerca de: ¿qué le motiva a participar del programa?, ¿qué significados tiene la espiritualidad?, ¿cómo cultiva la espiritualidad?, ¿cómo cuida su salud física?, ¿cómo cuida su salud mental?

Se contó con la aprobación por parte del Comité de Ética del Centro de Investigación de la Pontificia Universidad Javeriana. Las personas mayores firmaron el consentimiento informado, previa explicación del objetivo de investigación; se tuvieron en cuenta los criterios éticos mencionados en la Resolución 8430 de 1993 del Ministerio de Salud de Colombia, siendo una investigación sin riesgo, además de los mencionados en la Declaración de Helsinki.

La información recolectada durante el taller de arteterapia, cartas al cuerpo, diarios de campo y entrevistas, fue triangulada para la generación de categorías, y se procesó de acuerdo a la propuesta de Leininger y McFarland ${ }^{(21)}$. Primero, se dio lectura y relectura de las entrevistas, previa transcripción, así como a los diarios de campo, con el ánimo de familiarizarse con la información recolectada. Segundo, se identificó y precategorizó descriptores, así como sus componentes, teniendo en cuenta el dominio de interés de algunas preguntas. Tercero, 
se generaron patrones y análisis contextual, con el fin de encontrar la saturación de ideas y patrones recurrentes.

Finalmente, se identificaron categorías y subcategorías que fueran explicando las dimensiones del cuidado. Los pasos fueron flexibles y dinámicos, se contó con la revisión constante de los investigadores en el proceso, que permitió validar el procesamiento continuo de la información recogida, lo que aseguró la rigurosidad metodológica en el análisis, considerándose los criterios de credibilidad en tanto que los investigadores contaron con el juicio crítico de comprobación de las participantes, posibilitándose un espacio para contrastar con ellas las interpretaciones formuladas. En cuanto a la auditabilidad o confirmabilidad, se tuvo acceso a los registros de los diarios de campo, la grabación de las entrevistas y vídeos realizados durante las actividades. La transferibilidad se acoge en tanto que los investigadores dieron relevancia a los datos típicos de las participantes y la representatividad de los datos recolectados, desde una mirada de la totalidad. La identificación que se dio a las participantes de la investigación fue la siguiente: EP: entrevista participante y el número. Además, cartas al cuerpo y número de participante.

\section{RESULTADOS}

Datos sociodemográficos: Se entrevistaron 12 mujeres, personas mayores, en edades comprendidas entre los 66 a 81 años; 7 de procedencia rural, 4 de procedencia urbana y 1 de procedencia extranjera; 6 divorciadas, 5 viudas y 1 soltera; 9 recibían ingresos económicos derivados de pensión y 3 recibían apoyo económico proveniente de hijos o familiares. En relación con el nivel de estudios, se encontró que 1 obtuvo nivel profesional con posgrado, 6 profesionales universitarias, 1 sin culminar la carrera universitaria, 3 en nivel técnico y 1 con secundaria incompleta.

\section{Dimensión física, "Cuido mi cuerpo porque} en él guardo mi espíritu”: En esta dimensión, las personas mayores dan relevancia a integrar la capacitación permanente, la alimentación y el ejercicio. Desde allí es donde cuidan su espíritu. La visión que tienen del cuerpo está relacionada con lo espiritual, pues muchas de ellas consideran que deben cuidar el cuerpo porque en él se encuentra el espíritu de Dios:

"El cuidar mi cuerpo que es donde guardo mi espiritu, donde está mi alma; lo cuido con toda la parte de aseo, de estar lo mejor agradable para mi cuando me miro en el espejo" (EP 6). "Gracias amado cuerpo, mi cuerpo es la manifestación de Dios, mi creador, por lo tanto, doy perfecto y cada órgano de mi cuerpo como mis células, músculos, huesos tienen una función especifica que hace que mi cuerpo sea balanceado y bello" (Carta al cuerpo Participante 11).

La capacitación para prevenir daños al cuerpo y cuidarse mejor: El grupo de mujeres adultas mayores cuida de su salud a partir de la capacitación ofrecida por el programa al que asisten. Esto les permite tener conocimiento de sus propios procesos físicos, además, pueden aplicar los aprendizajes en sus prácticas cotidianas:

"A veces charlas, en donde, nos ponen, por ejemplo, la última charla fue muy buena, que fue: el sueño. Toda lo que era la fisiopatología del sueño, fue espectacular, con una persona muy profesional y nos dan Memoria; en memoria nos dan juegos" (EP 11).

Se encontró, además, que durante las charlas se establecen nuevos aprendizajes. Es una oportunidad que les permite resignificar aquellos aprendizajes del pasado, así como generar procesos de trasmisión de enseñanzas a otros:

"Una falla que tuvieron conmigo es que no nos enseñaron a tomar agua y la tomo, pero tengo que estar casi deshidratada para tomarme un vaso con agua... pienso que la lectura, el apoyo, las conferencias y todo lo que yo vea que me puedo enriquecer y que pueda además ayudar a alguien" (EP 7).

La capacitación incrementa los conocimientos para el autocuidado y coadyuva para el cuidado de la salud y atención integral a este grupo poblacional. Es visible el reconocimiento que las personas mayores dan a la formación y al acompañamiento profesional.

Comer sano mantiene el cuerpo en equilibrio y armonía: Para el cuidado físico se combinan prácticas de higiene personal con las relacionadas con el consumo de alimentos. Esto busca mantener el cuerpo en equilibrio. Es así como dicha dieta integra diariamente frutas, cereales y verduras, procurándose el consumo regular de agua que se asocia con mantener un bienestar general: 
"Cuidar no solamente en mi aseo sino en mi alimentación...consumo avena que es muy digestiva, las frutas porque me gustan y también porque me ayuda en la digestión... el agua me encanta porque sé que me ayuda" (EP 2).

Otro aspecto relevante, en la alimentación sana, radica en la capacidad de discernir entre alimentos buenos o malos. Ese conocimiento que se tiene de evitar en ciertas horas del día algunos alimentos como los derivados de lácteos, o de no excederse con el consumo de carne, harinas y grasas, son prácticas cotidianas que les permite tener el control de lo que consumen, atributo que refleja capacidad para la toma de decisiones, autonomía:

"No tomar ningún lácteo, nada de eso por la noche, sino cosas muy suaves" (EP 3). "Trato de que la comida sea balanceada, poca grasa y poca harina" (EP 7).

El ejercicio me hace sentir vivo: Con prácticas regulares como la gimnasia, el baile, el yoga, nadar o caminar, se genera percepción de bienestar físico y calidad de vida:

"Hacer gimnasia, hacer yoga, bailar" (EP 5). "Hacer ejercicio, caminar media hora todos los dias, eso es suficiente" (EP 5). "Hacer los ejercicios con el cuidado que requiere sin dañarme. Por ejemplo, no puedo hacer un ejercicio brusco si sé que me va a sentar mal, porque va a quedar adolorida" (EP 1). "¿cómo va de bonita, para dónde va? para el gimnasio, si señor así, pero con la ropa adecuada, mis tenis y todo adecuado" (EP 4).

2. Dimensión psicológica, "sentir y vivir con tranquilidad y en equilibrio me fortalece": Esta dimensión se conecta con aspectos que coinciden con la dimensión física y espiritual en cuanto a que aportan en la percepción de bienestar y vitalidad. Ese "estar bien" y sentir "tranquilidad", es favorable en el aumento del bienestar psicológico, mitigación de síntomas depresivos y de ansiedad.

Las buenas relaciones con los otros son fundamentales para estar bien: La asistencia al programa origina procesos de socialización, donde se logra interactuar con pares, compartir fechas especiales, dialogar sobre la vida y salir de la cotidianidad del hogar:

"Uno acá tiene buenas relaciones con las compañeras... contamos nuestras cosas...que el desayuno, que la fiesta, eso me ocupa, para no estar encerrada en la casa... es como la forma de aliviar uno sus problemas más que todo mentales" (EP 1). "Siento que me relaja mucho y que mi espiritu está feliz... el compartir, veo que mi espiritu se alegra" (EP 2).

La tranquilidad que se alimenta con la música y el encuentro con la naturaleza: Se expresan sentimientos favorables ante el contacto con la música y la naturaleza, estímulos externos que se vinculan a emociones positivas, facilitan el bienestar psicológico y físico, son recursos que posibilitan aliviarse de malestares:

"Para mi la música lo es todo, yo me levanto y pongo Cristovisión después pongo algo de noticias y después otra vez mi música...es la mejor compañia... la naturaleza me fascina, me gusta mucho la naturaleza" (EP 7).

Pensamientos positivos... "lo mejor para estar y sentirse bien": La capacidad de sentir vitalidad, de tomar decisiones, de incorporar aprendizajes nuevos y participar en diferentes actividades genera un bienestar mental. Para protegerse evitan pensamientos nocivos o la cercanía a estímulos como la televisión, que puedan generar emociones negativas, esto con la finalidad de no afectar el espíritu. No se refieren a la ingenuidad frente a las circunstancias por las cuales pasamos, sino tener la visión de "ver el vaso medio lleno, no medio vacío". Tener una mente positiva para ellas es clave a la hora de tener un cuidado espiritual:

"Sentirme que soy una persona activa, a pesar de mis años, para mi es importante, esa actitud de poder actuar, de aprender cosas nuevas, de participar en muchas cosas", "el estar relajada, el escuchar temas positivos, temas interesantes que ayuden a mejorar" (EP 2). "Psicológicamente, no dejarse llenar de todas esas noticias terribles que dan en la televisión y en la radio" (EP 5).

3. Dimensión espiritual, "la espiritualidad como un estilo de vida": Esta dimensión revela que no solo es importante ese "ser superior" en la vida de las personas mayores para su cuidado, sino que, además, se constata que la espiritualidad no se reduce a creencias religiosas, sino que se relaciona con otras prácticas que incentivan el mantenimiento de relaciones sociales positivas, un interés especial por el compromiso comunitario y el 
continuo crecimiento personal ${ }^{(8,22)}$

Creer en Dios, en los ángeles, en un ser superior, es la base de la espiritualidad: La espiritualidad está enmarcada en la capacidad de creer en un ser superior "Dios", a quien se le realizan oraciones para que provea todo. El asistir a la misa, comulgar y participar en otros sacramentos son acciones que permiten enfrentar la vida, las situaciones y mejorar el estado anímico. La creencia de la presencia de un ser superior se percibe como tener la compañía de alguien muy cercano:

"La base de todo es estar pegado de Dios" (EP 8). "Yo le digo a mis hijos, acójanse a Dios, miren que él es el único que lo puede guiar a uno, el único que le da las cosas" (EP 3). "Yo no rezo, yo oro, yo prefiero estar una hora, hablando con él, hablándole como mi amigo, yo siento que está al lado... yo me desahogo todos los dias" (EP 11).

La espiritualidad como servicio a los demás: La espiritualidad se materializa en prestar atención a las necesidades de otras personas, es la oportunidad de ayudar:

"Para mi la espiritualidad es el continuo crecer, el continuo escalar, el continuo evolucionar, el saber vivir la vida diaria y no saberla vivir yo, yo sólita sino con todos los que me rodean y con todos los seres bumanos" (EP 2).

Gratitud con Dios, con la vida, con uno mismo, es la base de la espiritualidad: La espiritualidad se cultiva con el agradecimiento a Dios, a las cosas que se tienen, por el tiempo presente, por la salud, por lo que se es capaz de hacer, las dificultades que se presentan y la presencia de seres queridos. Muchas de las participantes en este estudio manifiestan que ser una persona espiritual exige la gratitud hacia Dios por todo lo que nos dio, nos da y nos dará. Consideran incluso que el agradecimiento a Dios debe ser una actitud que nos acompañe durante todo el día:

"Para mi es la base fundamental, el que cree en Dios $y$ desde el momento que abre sus ojitos al otro día, debe darle gracias a Dios del nuevo amanecer, como al terminar el día darle gracias a Dios que todo terminó bien que no pasó nada, sino que él estuvo a todo momento cubriéndolo con su santísimo manto" (EP 6). "Le doy gracias a Dios del esposo que tuve, un hombre excepcional" (E 4). "Darle gracias a mi Dios y a mi virgencita que me ha dado una salud" (EP 5).
"El primer pensamiento para Dios dándole gracias por la noche de descanso, porque estoy viva, bendecir el dia por las dificultades" (EP 8).

\section{DISCUSIÓN}

En esta investigación se ha identificado la influencia de la espiritualidad en las dimensiones del cuidado en un grupo de mujeres, adultas mayores. Los hallazgos sociodemográficos presentan similitudes con otros estudios ${ }^{(11)}$ en cuanto a la mayor participación del género femenino en actividades lúdico-recreativas y rango de edad. Sin embargo, discrepa en relación con aspectos asociados a una posible fragilidad social puesto que las participantes del estudio en su mayoría refirieron tener formación académica, pensión y apoyo económico familiar $^{(8,11)}$.

La dimensión física está enmarcada en prácticas de cuidado alimenticio, capacitación y ejercicio, da cuenta de una percepción integral de armonía que se describe como esa posibilidad de mantenerse vivo, en actividades que producen placer, de apreciación de la propia vulnerabilidad, conciencia de un cuerpo que se deteriora, que requiere mantenerse, de su capacidad para recuperarse y de una espiritualidad que no se aparta del cuerpo, ni del bienestar psicológico ${ }^{(14)}$.

Cuidar el espíritu con la alimentación, la capacitación y el ejercicio, actúa favorablemente en las percepciones de vitalidad, de equilibrio y de autonomía. Además de una interacción social y profesional que visibiliza una relación óptima mente-cuerpo-espíritu, con el envejecimiento saludable. Así, se rompe el paradigma que relaciona la vejez con perspectiva de decadencia, y que se acerca más a una mirada positiva e integradora en las personas mayores ${ }^{(22)}$. Comer sano, para ellas, mantiene el cuerpo en armonía. Una dieta equilibrada, dentro de los procesos de cuidado integral de la salud, han sido mencionados en otros estudios para destacar la importancia que las personas mayores le dan a este aspecto en su envejecimiento saludable ${ }^{(23)}$. Las participantes reconocen que la adecuada alimentación, la capacitación y el ejercicio posibilitan lograr una armonía física, esa alimentación sana del cuerpo que es el reflejo del cuidado espiritual como se evidencia en otros estudios ${ }^{(8,23-25)}$. En otros estudios 
se menciona como "todo es bueno, pero no hay que abusar” partiéndose de la creencia de que así se obtiene equilibrio ${ }^{(8)}$.

Además, las mujeres, en el estudio, dan relevancia a su proceso de cuidado con la capacitación, apoyados por los profesionales que los acompañan; es así como configuran otros aprendizajes, tales como: el conocimiento de sí mismos y el cuidado propio en la edad en que se encuentran. Esto les ha permitido afrontar de manera constructiva y positiva sus cambios, orientarse a la socialización con sus pares y dialogar sobre su salud. Esto lo han mencionado estudios con población similar, en donde se muestra que la capacitación permite obtener una percepción de control a eventos de tipo externo y reportar beneficios personales ${ }^{(23)}$.

Los apoyos profesionales cobran poder, desde las aproximaciones educativas ${ }^{(11)}$, y el grupo tiene la posibilidad de mejorar su cuidado, de conocer el funcionamiento de su cuerpo y de entrenarse en procesos fundamentales como ha sido reportado en estudios con el mismo grupo poblacional ${ }^{(11)}$. Además, esto trae consigo satisfacciones de tipo emocional y psicológico puesto que contar con el apoyo de los profesionales se asocia con el mejoramiento de la autoestima, una actitud positiva en las actividades cotidianas y automotivación ${ }^{(8,11)}$.

Otros de los beneficios percibidos con la actividad física se describen como coadyuvantes para el mejoramiento de algunas dolencias o malestar físico, lo que también se ha evidenciado en otros estudios con personas mayores ${ }^{(23)}$, donde quienes físicamente son activos reportan mejoramiento en las actividades de la vida diaria, en los problemas de salud, en la capacidad de quemar calorías y en la dimensión psicológica, dado que pueden llegar a ser más optimistas y a mejorar el funcionamiento cognitivo. Prácticas como la gimnasia, el baile, el yoga o caminar les brinda bienestar físico y calidad de vida. Este hallazgo es similar en otros estudios donde la participación en la actividad física variada y acorde con los gustos e intereses de las personas mayores ejerce una influencia en la percepción de independencia funcional y bienestar subjetivo ${ }^{(22)}$. Las actividades físicas ayudan en la generación de sensaciones de vitalidad, permiten una percepción de vitalidad, distracción, mitigación de síntomas depresivos y fortalecimiento de la red social que se han tenido en cuenta en otros estudios para identificar patrones de cuidado y bienestar psicológico $^{(8,12,25)}$.

Por otra parte, llama la atención que las participantes dan cuenta de cuidados que se deben tener para la realización de los ejercicios y que se fundamentan en prestar atención para no excederse en la ejecución de los movimientos, así como de contar con el vestuario apropiado para realizarlo. Este aspecto ha sido identificado en otros estudios ${ }^{(11)}$ como algunos de los aportes de procesos formativos y de capacitación que se les brindan a las personas mayores, y que dan cuenta de la importancia de brindar atención de cuidado geriátrico integral para potenciar la autonomía y la autosuficiencia.

En la dimensión psicológica, las mujeres integran varios elementos importantes para el cuidado de su salud psicológica: la necesidad de contar con relaciones sociales, tener contacto con la música y con la naturaleza, para fomentar emociones positivas a través de pensamientos agradables, de esperanza y alegría. Esta dimensión está articulada con la anterior, pues un bienestar físico favorece uno psicológico. Esta relación ha sido reportada en otros estudios con población similar $^{(25,26)}$, donde el movimiento físico favorece aspectos psicológicos. Esa percepción de tener una vida plena se establece como elemento principal en la dimensión espiritual.

Tener una buena red social, compartir con el otro contribuye en la representación de ese bienestar y plenitud, lo que también alimenta el espíritu ${ }^{(27,}$ 28). Disponer de un recurso social posibilita el establecimiento de pautas en términos de roles sociales, mantenimiento de las sanas relaciones, mitigar sentimientos de soledad, aislamiento y abandono, factores protectores abordados en otros estudios $^{(27,29)}$.

La tranquilidad relacionada con la escucha de música y el encuentro con la naturaleza es otro elemento clave resaltado. Este hallazgo concuerda con estudios sobre espiritualidad ${ }^{(28)}$ que vinculan la armonía y el dominio con el medio ambiente como un recurso importante que aporta en la calidad de vida. Este nivel de autotrascendencia, denominado en algunos estudios como de tipo intrapersonal ${ }^{(29)}$, incide en las personas mayores en elevados indicadores de esperanza y plenitud que los lleva a estados de disfrute de la vida y conexión con el entorno que habitan.

En algunos estudios se ha revelado que pensamientos asociados con la aceptación del enve- 
jecimiento, encontrar significado a experiencias pasadas o aceptar la llegada de la muerte, se configuran en procesos de gerotrascendencia, donde a medida que las personas mayores envejecen, se conectan con mayor facilidad a pensamientos positivos que resignifican la vida y las experiencias vividas de acuerdo con su cultura, creencias y contexto $^{(28)}$.

Otros reportes ${ }^{(8,24)}$ han demostrado que en las personas mayores existe una negación frecuente a la autofocalización negativa ya una búsqueda optimista de solución a los problemas y a la reevaluación positiva. Esto coincide con lo reportado por los participantes del estudio, donde el cuidado de la salud se favorece por la conservación coherente entre emociones, sentimientos y pensamientos positivos. Para las participantes es fundamental vivir con tranquilidad, positivismo y alimentando el interior para potenciar todas las dimensiones de bienestar.

La dimensión espiritual se enmarca en una manera de vivir, influenciada por la creencia en un ser superior al que se le ofrecen diferentes prácticas, como mecanismos que visibilizan un contacto cercano ante ese ser. No todas las participantes se declararon católicas o de otra rama cristiana, sin embargo, sí manifestaron que la espiritualidad está referida a la creencia y relación con un ser superior. Estos hallazgos han sido identificados en otros estudios ${ }^{(15,29)}$ y se ha descrito como bienestar espiritual que identifica claramente unas prácticas $y$ creencias que desencadenan paz interior $y$ serenidad. Los mecanismos religiosos que hacen parte de la espiritualidad en algunos estudios ${ }^{(27)}$ han informado que estos son recursos valiosos que suscitan bienestar y un afrontamiento positivo ante la vida. El sentirse acompańado por Dios, tener su compañía y sus manifestaciones de amor, promueven de manera subjetiva una salud física y mental.

Otros reportes en la literatura ${ }^{(12)}$ enfatizan que las personas buscan constantemente el sentido a través de la relación con Dios y que esto se conecta con un entramado de creencias. Además, reconocen al cuerpo como un elemento temporal, así como los actos de fe y confianza que surten de significado a esa trascendencia de las personas desde una continua interacción cuerpo-mente, salud-enfermedad un equilibrio entre "el pensar, sentir y creer" ${ }^{(8)}$.

Esta creencia brinda la posibilidad de estar constantemente en función de ayudar a los otros y tener la experiencia constante del agradecimiento. Se puede dar múltiples formas de ayudar como el escuchar al otro, dar ayudas materiales, evitar las envidias, ofrecer una sonrisa, acciones que han sido reportadas en estudios sobre espiritualidad y cuidado donde también visibiliza una fuerte conexión entre prácticas de ayuda a otros, moralidad y religiosidad $^{(8)}$. Servir a los otros y el comportamiento altruista estrecha un lazo fuerte con la espiritualidad, el bienestar y la calidad de vida como se ha reportado en otros estudios ${ }^{(29)}$. Es un vínculo importante en la consolidación de la autotrascendencia desde un plano interpersonal, que si bien no supera la trascendencia intrapersonal, resulta importante para quienes creen en Dios o en un ser superior que los llama a estar al servicio de los demás.

Esa necesidad de trascendencia, de protección a la vida, se expresa en acciones de cuidado físico que repercuten en lo psicológico y viceversa, conectadas con la dimensión espiritual en tanto que hay una insistencia en la búsqueda de armonía entre la interacción personal, los otros y el entorno ${ }^{(23,25,30-}$ 31).

Con la aparición de esta dimensión en las personas mayores participantes del estudio, se vislumbra una estrategia de afrontamiento ante el cuidado, ya que aporta, en la cotidianidad, ante conflictos o circunstancias que surgen. Así mismo, las prácticas espirituales envuelven las otras dimensiones del cuidado para que sean más activas, preserven una visión positiva de la vejez y se mantenga una actitud de constante protección hacia la salud, hallazgos que coinciden con estudios con población similar ${ }^{(8,26,30)}$.

Asumir la vida con gratitud, sobre todo en esta parte de sus vidas, posibilita vivir con una actitud más positiva y lógicamente repercute en su estado físico y psicológico. Estos reportes son similares a otros estudios donde esta gratitud parte del principio de la creencia de Dios y que se expresa en agradecimiento hacia la presencia de familiares y amigos que los apoyan en el cuidado ${ }^{(8)}$ con la satisfacción de tener los medicamentos necesarios y la óptima alimentación, también como agradecimiento con la vida ${ }^{(31)}$, fijándose como un factor de protección para la salud mental y física ${ }^{(25,}$ 26).

La espiritualidad permea el cuidado, en tanto 
que refleja bienestar y armonía entre la interrelación con lo social, lo individual y el entorno ${ }^{(27)}$. Con las prácticas espirituales, se abre paso al disfrute de la vida, al agradecimiento constante, a esa necesidad y posibilidad de ayudar a otros, lo que sugiere que las dimensiones física y psicológica se ven influenciadas por este bienestar ${ }^{(29)}$.

Los resultados reflejan que la dimensión espiritual es la base del cuidado, la posibilidad de creer en un ser superior, "Dios", que ayuda a alcanzar un estado de bienestar. A partir de prácticas que los acercan a ese ser que ayuda en todos los aspectos de la vida, los aportes de la espiritualidad al cuidado sugieren que esta se configura en un elemento sanador, hallazgo documentado en otros reportes ${ }^{(8,12,14,24)}$.

Las construcciones del cuidado, desde las dimensiones estudiadas, dan cuenta de un proceso de envejecimiento placentero, positivo, de una constante reinvención humana que no desconoce la diversidad cultural que se nutre del acompañamiento profesional, social y espiritual en esa búsqueda constante de satisfacción de la vida ${ }^{(23)}$.

El estudio resalta la importancia de reconocer los aspectos del cuidado espiritual en las mujeres adultas mayores y fortalecerla mediante la integración de estrategias de intervención cultural en salud acordes a una visión que integra a la espiritualidad como el escenario para el desarrollo integral de las personas mayores $^{(27)}$.

Se resalta la importancia de estudiar las dimensiones del cuidado desde la perspectiva espiritual en hombres adultos mayores, quienes menormente participan en procesos formativos de promoción de la salud ${ }^{(10,11)}$, además de los aspectos relacionados con la adherencia de los mismos a procesos de formación en el cuidado de la salud, con la intención de proporcionar estrategias acordes a su cosmovisión y a la construcción de género inmersa en su cultura.

\section{CONCLUSIONES}

Se evidenció que para las mujeres que participaron en la investigación, la espiritualidad les brinda una perspectiva importante en sus vidas y en las distintas dimensiones del cuidado. En una etapa de su existencia en que algunos considerarían como de descanso, ellas encuentran que esta dimensión sigue proporcionándoles sentido y sienten que se les permite ser más resiliente.

En muchas de las entrevistas que se hicieron se manifestó que la espiritualidad ayuda a vivir los momentos difíciles o las enfermedades. Alimentar el espíritu ayuda a sobrellevar o vivir de otra manera la realidad, porque somos un cuerpo que se va envejeciendo, entonces se convierte en un acicate para vivir con más alegría.

Para ellas, la espiritualidad es una parte esencial de los seres humanos e implica todo un estilo de vida. Si asumimos nuestra existencia de esta manera repercutirá positivamente en todos los otros niveles de nuestra existencia. Además, las dimensiones de lo físico, psicológico y espiritual, deben estar articuladas y, por ende, deben cuidarse en esos tres niveles. Es un eje transversal a todos los niveles de la vida y un elemento integrador.

Estos hallazgos son un reto para la formulación de políticas públicas, las organizaciones y los profesionales de la salud, para la consolidación de diagnósticos, protocolos e intervenciones que garanticen una atención integral.

\section{REFERENCIAS}

1. Organización Mundial de la Salud. Informe mundial sobre el envejecimiento y la salud [Internet]. 2015 [citado 15 nov 2019]. Disponible en: https://apps. who.int/iris/bitstream/handle/10665/186466/ 9789240694873_spa.pdf

2. Ministerio de Salud y Protección Social. Envejecimiento demográfico. Colombia 1951-2020. Dinámica demográfica y estructuras poblacionales [Internet]. 2013 [citado 15 feb 2020]. Disponible en: https://www.minsalud.gov.co/sites/rid/Lists/ BibliotecaDigital/RIDE/DE/PS/Envejecimientodemografico-Colombia-1951-2020.pdf

3. Esmeraldas VE, Falcones MR, Vásquez MG, Solórzano JA. El envejecimiento del adulto mayor y sus principales características. RECIMUNDO [Internet]. 2019 [citado 16 nov 2019]; 3(1): 5874. Disponible en: https://doi.org/10.26820/reci mundo/3.(1).enero.2019.58-74

4. Calvo-Soto AP, Gómez-Ramírez E. Condiciones de salud y factores relacionados con discapacidad en adultos mayores - Una reflexión para la atención. Univ Salud [Internet]. 2018 [citado 16 nov 2019]; 20(3): 270-282. Disponible en: https://doi. org/10.22267/rus.182003.131

5. Purnell LD. El modelo de competencia cultural 
de Purnell: Descripción y uso en la práctica, educación, administración e investigación. Cultura de los Cuidados [Internet]. 1999 [citado 17 nov 2019]; 3(6): 91-97. Disponible en: https://doi. org/10.14198/cuid.1999.6.13

6. Escobar-Castellanos B, Paravic-Klijn T. La transculturalidad, elemento esencial para mejorar la atención en salud y de enfermería. Enfermería Actual de Costa Rica [Internet]. 2017 [citado 18 nov 2019]; 32. Disponible en: http://dx.doi. org/10.15517/revenf.v0i33.29627

7. Naranjo-Hernández Y, Sánchez-Carmenate M, Pérez Y. Estrategia de cuidado de los adultos mayores hipertensos en la comunidad. Rev cuba enferm [Internet]. 2019 [citado 20 nov 2019]; 35(3): 1-15. Disponible en: http://www.revenfermeria.sld.cu/ index.php/enf/article/view/2670/462

8. Vargas-Santillán ML, Arana-Gómez B, GarcíaHernández ML, Ruelas-González G, MelguizoHerrera E, Ruiz-Martínez AO. Prácticas de autocuidado en adultos mayores: un estudio cualitativo en una población mexicana. Rev Enf Ref [Internet]. 2018 [citado 21 nov 2019]; IV(16): 117-126. Disponible en: https://doi.org/10.12707/ RIV17065

9. Galvis-Palacios LF, López-Díaz L, Velásquez V. Patrones culturales de cuidado familiar al adulto mayor en condición de discapacidad y pobreza. Index Enferm [Internet]. 2018 [citado 22 nov 2019]; 27(3): 170-174. Disponible en: http:// scielo.isciii.es/scielo.php?script=sci_arttext\&pid $=$ S1132-12962018000200013

10. Muñoz-Muñoz CG. El juego como recurso terapéutico en la intervención comunitaria con personas mayores. Humanidades Médicas [Internet]. 2016 [citado 13 abr 2021]; 16(1): 84-97. Disponible en: http://scielo.sld.cu/pdf/hmc/v16n1/hmc 06116.pdf

11. Puello-Alcocer EC, Amador-Ahumada C, OrtegaMontes JE. Impacto de las acciones de un programa de enfermería con enfoque promocional y de autocuidado en la capacidad funcional de adultos mayores. Rev Univ Salud [Internet]. 2017 [citado 18 dic 2019]; 19(2): 152-162. Disponible en: https://doi.org/10.22267/rus.171902.78.

12. Palacio-Vargas CJ. La espiritualidad como medio de desarrollo humano. Cuest Teol Filos [Internet]. 2015 [citado 24 nov 2019]; 42(98): 459-481. Disponible en: https://revistas.upb.edu.co/index. $\mathrm{php} /$ cuestiones/article/view/251

13. Pérez-Hernández $S$, Okino-Sawada $\mathrm{N}$, DíazOviedo A, Lordelo-Marinho PM, Ruiz-Paloalto ML. Espiritualidad y calidad de vida en mujeres con cáncer de mama: una revisión integrativa. Enferm Univ [Internet]. 2019 [citado 25 nov
2019]; 16(2): 185-195. Disponible en: https://doi. org/10.22201/eneo.23958421e.2019.2.643

14. Becerra-Melo, S. La espiritualidad en pacientes que viven con VIH. Cuest Teol [Internet]. 2018 [citado 25 nov 2019]; 45(103): 149-175. Disponible en: https://doi.org/10.18566/cueteo.v45n103.a07

15. Ledesma-Rivera A, Montero M. Espiritualidad y religiosidad en adultos mayores mexicanos. Salud Mental [Internet]. 2005 [citado 27 nov 2019]; 28(6): 51-58. Disponible en: http://www. revistasaludmental.mx/index.php/salud_mental/ article/view/1081

16. Longuiniere AC, Yarid SD, Silva EC. Influência da religiosidade/espiritualidade do profissional de saúde no cuidado ao paciente crítico. Rev Cuidarte [Internet]. 2018 [citado 28 nov 2019]; 9(1): 19611972. Disponible en: https://doi.org/10.15649/ cuidarte.v9i1.413.

17. Ross L, Miles J. Spirituality in heart failure: A review of the literature from 2014 to 2019 to identify spiritual care needs and spiritual interventions. Curr Opin Support Palliat Care [Internet]; 2019; [citado 5 dic 2019]; 14 (1): 9-18. Disponible en: https://doi.org/10.1097/SPC.0000000000000475

18. Rosas-Jiménez CA, Díaz-Díaz Á. La escucha: elemento fundamental en la atención espiritual del cuidado paliativo. Persona y bioética [Internet]. 2017 [citado 10 dic 2019]; 21(2): 280-291. Disponible en: https://personaybioetica.unisabana. edu.co/index.php/personaybioetica/article/ view/7877

19. Páramo-Morales D. La teoría fundamentada (Grounded Theory), metodología cualitativa de investigación científica. Pensam gest [Internet]. 2015 [citado 10 dic 2019]; 39: 119-146. Disponible en: http://www.scielo.org.co/scielo.php?script=sci_ arttext\&pid=S1657-62762015000200001

20. Guber S. El salvaje metropolitano. Reconstrucción del conocimiento social en el trabajo de campo. Buenos Aires: Paidós; 2005. 220 p. Disponible en: http://www.derechoshumanos.unlp.edu.ar/assets/ files/documentos/el-salvaje-metropolitano.pdf

21. Leininger $M, M c F a r l a n d ~ M$. Transcultural nursing: concepts, theories, research and practice, $3^{\mathrm{a}} \mathrm{ed}$. New York, McGraw-Hill; 2002. 648 p.

22. Wittenberg E, Ragan SL, Ferrell B. Exploring Nurse Communication About Spirituality. Am J Hosp Palliat Care [Internet]. 2017 [citado 20 ene 2020]; 34(6): 566-571. Disponible en: https://doi. org/10.1177/1049909116641630

23. Martín-Aranda R. Actividad física y calidad de vida en el adulto mayor. Una revisión narrativa. Rev haban cienc méd [Internet]. 2018 [citado 21 ene 2020]; 17(5): 813-825. Disponible en: http:// www.revhabanera.sld.cu/index.php/rhab/article/ 
view/2418

24. Alberola S, Oliver A, Tomás JM. Validación de un modelo intercultural de envejecimiento exitoso en población espańola. Gerokomos [Internet]. 2017 [citado 21 ene 2020] 28(2): 63-67. Disponible en: http://scielo.isciii.es/scielo.php?script=sci_abstract \&pid=S1134-928X2017000200063

25. Noriega C, Velasco C, Pérez-Rojo G, Carretero I, Chulián A, López J. Calidad de Vida, Bienestar Psicológico y Valores en Personas Mayores. Revista Clínica Contemporánea [Internet]. 2017 [citado 24 ene 2020]; 8(e1): 1-13. Disponible en: https:// doi.org/10.5093/cc2017a1

26. Aguilera-Velasco M, Pérez-Solís J. Revisión bibliográfica sobre el bienestar en adultos mayores jubilados. Revista Iberoamericana de Psicología [Internet]. 2016 [citado 25 ene 2020]. 9(2): 43-59. Disponible en: https://reviberopsicologia.ibero. edu.co/article/view/rip.9204

27. Gallardo-Peralta LP, Cuadra-Peralta A, VelosoBesio C. Validación de un índice breve de religiosidad y espiritualidad en personas mayores. Revista de Psicología [Internet]. 2018 [citado 2 feb 2020]; 27(1): 1-13. Disponible en: http://dx.doi. org/10.5354/0719-0581.2018.50736
28. Pilger-Calíope SRO, Lentsck-Maicon H, Marques $S$, Kusumota L. Spiritual well-being and quality of life of older adults in hemodialysis. Rev Bras Enferm [Internet]. 2017 [citado 2 feb 2020]; 70(4): 689-696. Disponible en: http://dx.doi. org/10.1590/0034-7167-2017-0006

29. Guerrero-Castañeda RF, Lara-Pérez R. Nivel de autotrascendencia en un grupo de adultos mayores mexicanos. Rev Cuid [Internet]. 2017 [citado $4 \mathrm{feb}$ 2020]; 8(1): 1476-1487. Disponible en: https:// doi.org/10.15649/cuidarte.v8i1.354

30. Alvarado-Valle E, Rodríguez-Jiménez S, CárdenasJiménez M, Pacheco-Arce AL. El cuidado espiritual un camino para trascender en la razón de ser de la Enfermería. Rev Enferm Inst Mex Seguro Soc [Internet]. 2018 [citado 2 feb 2020]; 26(1): 56-61. Disponible en: https://www.medigraphic.com/cgibin/new/resumen.cgi?IDARTICULO=78915

31. De Alba GM. Representaciones sociales y experiencias de vida cotidiana de los ancianos en la Ciudad de México. Estud demogr urbanos [Internet]. 2017; [citado 2 feb 2020]; 32(1): 9-36. Disponible en: http://www.scielo.org.mx/scielo. php?script $=$ sci_arttext $\&$ pid $=$ S0186-72102017000 100009 